\title{
Interactive comment on "On modelling the kinematics and evolutionary properties of pressure pulse driven impulsive solar jets" by Balveer Singh et al.
}

\section{Anonymous Referee \#1}

Received and published: 23 May 2019

This work deals with the parametric study of the pressure pulse driven solar jets in the chromosphere. The work involves realistic solar atmosphere and magnetic field mimicking the open and gravitationally stratified open field magnetoplasma system of the quiet-Sun atmosphere. The model is detailed study of the kinematics of the cool chromospheric jets which could be generated by the localized heating and the pressure perturbations. This model extends the previous works on the pressure pulsle driven spicules to a more general cases applied for the variety of the solar jets like marcospicules, network jets, cool jets, confined surges etc with the application of realistic solar atmosphere and temperature profile. The implementation of Transition Region also brings the more appropriate results. Paper deals with the kinematical properties 
and morphological evolutions of the cool jets that can be applicable to the variety of chromospheric and TR jets. The paper is clearly written and basic results are presented very well. I do not offer any major revision and recommend the paper for its publications. However, authors should explain/implement some minor comments before its final publication.

(1) In the introduction, author should include/discuss some more works that are focused on "pressure pulse driven solar jets", e.g., Martinez-sykora et al. 2011, Judge et al. 2012, Kuzma et al. 2017

(2) Explain why you specifically chosen certain height for pressure pulse?

(3) Why the particular pulse is chosen?

(4) Please describe a little more about the response of chromosphere pressure disturbance.

(5) What is the physical implication of chosen magnetic field?

(6) Describe a little more specifically that how pressure driven jets are more likely in the chromsophere than other wave driven jets.

Apart from these comments, Editor may also suggest the authors to improve the English of the paper at some stage.

Interactive comment on Ann. Geophys. Discuss., https://doi.org/10.5194/angeo-2019-67, 2019. 